\title{
Stability of Amorphous Structures with Voids
}

\author{
Charanbir kaur and Shankar P. Das \\ School of Physical Sciences, \\ Jawaharlal Nehru University, \\ New Delhi 110067, India.
}

\begin{abstract}
We incorporate the role of free volume in the density function of the amorphous structure and study its effects on the stability of such structures. The Density Functional Theory is used to explore this "Free Volume Model" of the supercooled structures. The Free energy minimization is carried out using the void concentration as a variational parameter. A critical value of this concentration exists corresponding to the Free energy minima of the amorphous structure. An increase in the stability is observed due to the inclusion of voids in the density structure. This study is conducted for both the weakly and highly localized amorphous structures. The free volume concentration shows a power law decrease with density for the weakly localized states and a linear decrease for the highly localized amorphous structures.
\end{abstract}

PACS number(s) : 64.70Pf, $64.60 \mathrm{Cn}, 64.70 \mathrm{Dv}$ 


\section{Introduction}

The transformation of a continuum ergodic liquid into an amorphous glassy state with solidlike behaviour has been an area of much current research interest. The ambiguities concerning the glassy or the supercooled state have led to the development of a large number of theoretical approaches [1, 2, 3, 4] that are used to study the basic characteristics of a system undergoing such a transition. These techniques explore both the dynamic and the thermodynamic properties of the supercooled states. A liquid is supercooled while avoiding crystallization such that its density structure loses the homogeneity of a liquid state and attains a heterogeneous density profile. From a thermodynamic point of view, the supercooled state represents a metastable state relative to the crystal and is characterized by an inhomogeneous and aperiodic density structure. This aperiodic crystal picture of the supercooled state is considered here to study its stability from a thermodynamic approach. In this work, we intend to explore the "Free Volume" picture of the supercooled liquid [1] [5] by studying the Free energy landscape of an amorphous lattice characterized by a finite fraction of vacancies.

Fox and Flory [6] first postulated that the liquid-glass transition resulted from the decrease of "free volume" of the amorphous phase below some critical value. Subsequently, this concept was further elaborated by Cohen \& Turnbull [1] and later by Cohen \& Grest [5] who formally developed the theoretical aspects using the percolation theory. The basic phenomenology behind the Free Volume models is that transport of particles occurs by their flow into voids of a size greater than a critical value formed by the redistribution of some "free volume". The latter is loosely defined as some surplus volume that is not taken up by the particles. As a consequence of this theory, the relation between the free volume con-

centration and the transport properties of the supercooled state like the viscosity was also investigated. Doolittle [7] found empirically that the fluidity (inverse of viscosity) of many 
simple hydrocarbon liquids could be represented in terms of free volume as,

$$
\phi=\phi_{o} \exp \left(-\frac{c v_{o}}{v_{f}}\right)
$$

where $\phi$ denotes the fluidity and $c$ is a constant of order unity. Here $v_{f}$ denotes the average free volume per molecule, $v_{f}=\bar{v}-v_{o}, \bar{v}$ being the average volume per molecule and $v_{o}$ is the van der Waals volume of the molecule. Williams etal. showed that this result is valid for a large number of glass formers. They proposed that a linear relation exists between the free volume $v_{f}$ and the distance from transition on the temperature scale $T-T_{g}$. This, through eqn.(11), in turn gives rise to the typical Vogal-Fulcher divergence of viscosity.

The Density Functional Theory (DFT) is an appropriate liquid based theory to undertake this study. This theory is based upon the simple ideology of identifying a solid as a strongly nonuniform liquid. This notion of describing the properties of a highly non-uniform phase in terms of the fairly well-developed liquid state theory was first put forward by Kirkwood and Monroe [8]. Later on, Ramakrishnan and Yussouf (RY) [9] formulated it in terms of direct correlation function and successfully applied this concept to liquid-solid coexistence problem by expressing the properties of the highly organized solid( the non-uniform phase) as a perturbation of those of the coexisting liquid. The mathematical formulation of this simple concept was further elucidated by Haymet and Oxtoby [10] and a fair amount of success has since been achieved in solving problems related to simple inhomogeneous density structures. This theory has been lately applied to study the static aspects of supercooled amorphous structures. These studies were mainly initiated by Singh et.al. [3] and were followed by similar studies using different density functional treatments [11, 12]. Two types of Free energy minima were located for such systems. The first corresponding to highly localized amorphous structures that were identified as the "hard sphere glass" state [3, 11, 12]. A different class of minima were detected [13] in the Free energy landscape of the same random structure, that represented weakly localized heterogeneous structures. These structures were shown to be more realistically associated with the structure of supercooled states seen in 
computer simulation studies. The metastable character of the different type of minima in the free energy landscape by modeling "free volume" in the density function characteristic of these structures is explored here. This has the effect of increased heterogeneous character of the underlying amorphous structure. We compute the Free energy functional using the RY expansion around a uniform state.

The motivation of the present work is to formulate the concept of free volumes in a simple density functional formalism and use its variational nature to investigate whether there is any optimum value of the free volumes associated with the metastable phase, giving a free energy minima. In the model presented here, the free volumes in the system are constructed as the vacancies at the prescribed lattice sites of the Bernal's random structure [14. Such a modeling leads to a scenario of increased amount of local unoccupied volume in the system and that is how we define "free volume" in this work. The major requirement of such theories is the formulation of the test density function which is relevant to the type of structure whose stability is being considered. The variational principle is then invoked to determine the density structure corresponding to the minimum of the Free energy functional. P. Tarazona [15] first proposed to consider the ensemble averaged density distribution as a summation over Gaussian profiles centered over the lattice sites of the given inhomogeneous structure. Since then, such a formulation has been followed almost uniformly in all the related Density Functional studies. In this approach, the density is parameterized in terms of the mass localization parameter $\alpha$ which is proportional to the width of the Gaussian profiles. This parameter has the effect of quantifying the motion of particles in the system. The $\alpha \rightarrow 0$ limit depicts Gaussian profiles of infinite width and thus the corresponding phase represents the homogeneous liquid state. Increasing values of $\alpha$ represent increasingly localized structures and thus referring to greater inhomogeneity in the system. The $\alpha$ corresponding to the Free energy minima finally determines the phase which the system prefers thermodynamically. To include vacancies in the system, the parameter space of the density function is increased. 
The homogeneous presence of a specific concentration of vacancies in the system leads to a uniform decrease in the number of occupied sites in each co-ordination shell around any arbitrary position in the system. In an ensemble-averaged picture this amounts to expressing the density function as,

$$
\left.\rho(\vec{r})=\mathcal{P}\left(\frac{\alpha}{\pi}\right)^{\frac{3}{2}} \sum_{i=1}^{N_{L}} e^{-\alpha\left|\vec{r}-\vec{R}_{i}\right|^{2}}\right)
$$

where $\mathcal{P}$ actually serves as the fraction of sites present in any $i^{t h}$ shell around the position $\vec{r}$ in the system, such that $\Delta_{v}=1-\mathcal{P}$ represents the vacancy concentration in the system. We assume the vacancies to be distributed homogeneously throughout the system and hence this fraction is the same for all the shells around any arbitrary position in the system. This approximation is followed from Jones and Mohanty [16] who considered defects in a perfect crystal structure. Here $N_{L}$ is the number of lattice sites in volume $\mathrm{V}$ out of which $N$ sites are occupied by the particles. The average particle density is thus, $\rho_{o}=\mathcal{P} \rho_{b}$ where $\rho_{b}$ represents the average lattice density. Thus the Free energy minimization is carried out with respect to $\alpha$ and $\mathcal{P}$. These two parameters elaborate on two different structural aspects of the stable phase. We mainly explore the existence of a critical free volume concentration $\mathcal{P}$ and its variation with density for the two different class of minima seen for amorphous structures.

The paper is organized in the following manner : In section II we give the details related to the calculation of the Free energy functional formulated so as to include the effects of free volume. In section III we evaluate the effects on the stability of the amorphous structures by the induction of vacancies in the density structure. Here we present the analysis conducted for amorphous system in both the extremely localized and the weakly localized regimes. The implications of these results are further discussed in the section IV and relevance with the dynamical studies is quantitavely explored. 


\section{The Model Studied}

The Helmholtz Free energy can be expressed as a sum of the purely entropic contribution given by the ideal gas term and the contribution due to the interactions in the system identified as the excess term in the general terminology.

$$
\mathcal{F}[\rho(\vec{r})]=\int d \vec{r} \rho(\vec{r})\left(\ln \left(\wedge^{3} \rho(\vec{r})\right)-1\right)-\Phi[\rho(\vec{r})]
$$

Here $\rho(\vec{r})$ is the ensemble averaged local density distribution and $\Phi$ represents the excess part of the Free energy. $\Phi[\rho(\vec{r})]$ is expressed as a perturbation around a liquid of density $\rho_{o}$. The functional Taylor series expansion about the homogenous density distribution $\rho_{o}$ is given as [9],

$$
\begin{aligned}
\Phi[\rho(\vec{r})]-\Phi\left(\rho_{o}\right)= & \int d \vec{r}_{1} \delta \rho\left(\vec{r}_{1}\right)\left[-\beta \mu_{o}+\ln \left(\wedge^{3} \rho_{o}\right)\right] \\
& +\frac{1}{2} \int d \vec{r}_{1} \int d \vec{r}_{2} c\left(\vec{r}_{1}-\vec{r}_{2} ; \rho_{o}\right) \delta \rho\left(\vec{r}_{1}\right) \delta \rho\left(\vec{r}_{2}\right)
\end{aligned}
$$

where $\mu_{o}$ is the chemical potential of the uniform phase and $c\left(r ; \rho_{o}\right)$ is the direct correlation function of the homogeneous state of density $\rho_{o}$. In the present work we consider the perturbation expansion of the excess Free energy around the uniform system of the same density as the average density of the corresponding inhomogeneous structure, i.e.,

$$
\rho_{o}=\frac{1}{V} \int d \vec{r} \rho(\vec{r})
$$

The Free energy expression in this case reduces to the following form,

$$
\begin{aligned}
\mathcal{F}[\rho(\vec{r})]-\mathcal{F}\left(\rho_{o}\right)= & \int d \vec{r} \rho(\vec{r}) \ln \left(\frac{\rho(\vec{r})}{\rho_{o}}\right) \\
& -\frac{1}{2} \int d \vec{r}_{1} \int d \vec{r}_{2} \delta \rho\left(\vec{r}_{1}\right) \delta \rho\left(\vec{r}_{2}\right) c\left(\vec{r}_{1}-\vec{r}_{2} ; \rho_{o}\right) .
\end{aligned}
$$

Here $\delta \rho(\vec{r})$ is the deviation of the inhomogeneous state density $\rho(\vec{r})$ from the average density $\rho_{o}$. The minimization of this free energy functional then determines the density function corresponding to the stable ( or metastable) state of the given system. The first term is 
the purely entropic contribution to the Free energy and the interactions in the system are responsible for the second term.

This functional is then minimized in a constrained manner by the specification of the underlying lattice as the input. This information is carried by the site-site correlation function $g(R)$ of the particular structure considered. Bernal's random structure [14] is considered as a good approximation for the static structure of undercooled liquid state. We follow the earlier approach of supplying the $g(R)$ of the Bernal structure determined from the Bennett's algorithm [17] and approximate [1],

$$
g(\vec{R})=g_{B}\left[R\left(\frac{\eta}{\eta_{o}}\right)^{\frac{1}{3}}\right]
$$

where $\eta$ denotes the average packing fraction. Here $\eta_{o}$ is used as a scaling parameter for the structure, [11, 12 such that at $\eta=\eta_{o}$ the structure corresponds to Bernal packing, so that different values of $\eta_{o}$ relate to different structures. Now we elaborate the computational aspects of reducing these expressions for the specific case of a random structure.

With the density function expressed in terms of defects, i.e. eqn.(2), the Ideal gas part of the Free energy per particle can in general be expressed as,

$$
\Delta f_{i d}[\rho]=\mathcal{P} \int d \vec{r} \phi(\vec{r})\left[\ln \left(\frac{\mathcal{P}}{\rho_{o}} \int d \vec{R} \phi(\vec{r}-\vec{R})\left(\delta(\vec{R})+\rho_{o} g(\vec{R})\right)\right)\right]
$$

for an amorphous structure described by the radial distribution function $g(R)$ referred above. This is obtained by the use of eqn.(2) in the ideal gas term of eqn.(河). The random structure is marked by a vacancy concentration of $\Delta_{v}$. In the above expression, the function $\phi(\vec{r}-\vec{R})$ represents a Gaussian function centered at a position $\vec{R}$, i.e., $\phi(\vec{r}-\vec{R})=\left(\frac{\alpha}{\pi}\right)^{\frac{3}{2}} e^{\left.-\alpha|\vec{r}-\vec{R}|^{2}\right)}$. The density structure of the system in a highly localized state is represented by sharply peaked Gaussian functions centered around the lattice sites. This asymptotic limit corresponds to large $\alpha$ and the ideal gas part of free energy (eqn.(8)) reduces to,

$$
\Delta f_{i d}[\rho] \approx-\left[\frac{3}{2}+\ln \rho_{o}-\ln \mathcal{P}+\frac{3}{2} \ln \left(\frac{\pi}{\alpha}\right)\right]
$$


This is obtained by replacing the summation inside the logarithmic term by just the contribution from the nearest site. This reduction is although not valid for consideration of the weakly localized state of the amorphous structure where the $\alpha$ values are small and the Gaussian functions wide enough to cause considerable overlap over different sites such that the above reduction can no longer be justified. Thus in the range of small $\alpha$ we numerically evaluate the equation (8) as such and the equation (9) in the large $\alpha$ regions. In our earlier work [13] we have shown that these two expressions start merging for typically $\alpha>40$ within 1\%. Similarly we formulate the evaluation of the excess term per particle as,

$$
\begin{aligned}
\Delta f_{\text {ex }}= & \rho_{o} \int d \vec{r} c\left(r ; \rho_{o}\right)-\frac{\mathcal{P}}{2} \int d \vec{r}_{1} \int d \vec{r}_{2} \phi\left(\vec{r}_{1}\right) \phi\left(\vec{r}_{2}\right) c\left(\vec{r}_{1}-\vec{r}_{2} ; \rho_{o}\right) \\
& -\frac{\mathcal{P}}{2} \int d \vec{R} \int d \vec{r}_{1} \int d \vec{r}_{2} \phi\left(\vec{r}_{1}\right) \phi\left(\vec{r}_{2}-\vec{R}\right)\left(\delta(\vec{R})+\rho_{o} g(\vec{R})\right) c\left(\vec{r}_{1}-\vec{r}_{2} ; \rho_{o}\right)
\end{aligned}
$$

Here the direct correlation function, $c(r)$, carries the liquid state interaction information. For the description of $c(r)$ we have used the Percus-Yevick (PY) form with Verlet-Weiss correction as obtained by Henderson and Grundke [18, 19]. This concludes the formulation of the free energy functional used to measure the effects of free volume on the stability of amorphous systems.

\section{Stability of the amorphous structure}

We now elaborate on the methodology used to investigate the stability of the amorphous structure characterized by a finite vacancy fraction $\Delta_{v}$. The free-energy functional is numerically evaluated using the expressions (8) to (10) at a given average density $\rho_{o}$. The parameters $\alpha$ and $\mathcal{P}$ are varied simultaneously and two minima are detected in the two di-

mensional $(\alpha, \mathcal{P})$ free energy landscape. One corresponds to the weakly localized state of the amorphous structure since it is found corresponding to low $\alpha$ values. The other corresponds to very highly localized structures characterized by large $\alpha$ values. We illustrate the minimum on the Free energy landscape with respect to the variational parameter $\mathcal{P}$ in the 
Figure 1. The plotted values correspond to the minima in the large $\alpha$ space. The minimum value of the Free energy is observed at $\mathcal{P}=0.951$ at density $\rho_{o}=1.12$, as indicated by the arrow. This figure depicts a considerable increase in the stability of the structure by the induction of free volumes in the structure. The $\Delta f$ values decreases from $1.64(\mathcal{P}=1)$ to -0.44 due to the presence of free volume in the system. As an inset in this figure, the $\alpha$ space minimization is shown at the same density. In Figure 2 we show the corresponding curve for the weakly localized structure at the same density. The $\alpha$-space variation is shown as an inset in this figure where each value corresponds to the vacancy concentration obtained as a partial minimum in the $\mathcal{P}$ space. In this case the optimum value of $\mathcal{P}$ is obtained at 0.946 , corresponding $\Delta f$ value being -1.325 . The effect of vacancies on the stability of the weakly localized structure is not as significant as that observed for the highly localized structures. This is because in the weakly localized structures the Gaussian profiles of the particles are already too smeared. The vacancies are not expected to alter the density structure to a large extent and hence the free energy values do not change by a large percentage. Nevertheless, the existence of a minimum in the $\mathcal{P}$ space at a value lesser than one indicates the thermodynamic preference of the amorphous state for a structure characterized by a homogeneous presence of vacancies and thus establishing the "free-volume" picture of supercooled states from a density functional approach.

The two class of minima observed in amorphous structures represent two different stationary states of the system and the dynamics of the system should show different characteristics around these minima. This is indicated by the two different type of behavior shown by the variation of vacancy concentration with respect to the average density. It follows a linear relation with the average particle density of the highly localized amorphous structure. This is shown in the Figure 3, where the solid line is the linear curve fitting to the variation of $\mathcal{P}$ versus $\rho_{o}$ for the state corresponding to the high $\alpha$ minimum. This predicts that the system will completely freeze, i.e., the free volume concentration will vanish, at packing fraction 
$\eta=.62$. The Figure 4 illustrates the corresponding curve for the weakly localized structure, corresponding to the lower width parameter values. The free volume concentration shows a power law decrease with the increase in the density. The solid line of the curve depicts this functional variation that shows the divergence at $\eta=.58$ with an exponent .128 .

In Figure 5 we have shown the variation of the free energy versus the average density for both the highly localized and the weakly localized amorphous structures. The metastability of the highly localized amorphous structure starts coming close to that of the weakly localized state at higher densities due to the induction of free volumes in the structure. The highly localized state starts becoming more stable than the homogenous state after density $\rho_{o}=$ 1.12. In the similar density structures considered without defects this density has been reported to be 1.14 [3]. The Figure 6 illustrates the variation of the $\alpha$ with $\mathcal{P}$ at density $\rho_{o}=1.08$. This $\alpha$ corresponds to the free energy minima at a given value of the defect concentration $\mathcal{P}$. The monotonic decrease depicts a preference for decreasing localization in the system with decrease in defects concentration $\Delta_{v}$.

\section{Discussion}

We have investigated various features of the supercooled states by considering the presence of a finite void concentration in an amorphous lattice. In formulating the test density function of the amorphous structure we have basically extended the idea used by Jones and Mohanty [16], who considered defects in crystal structures. By taking our cue from the Free Volume Theory, we have formulated the corresponding theory in a Density Functional Formalism. Important implications are drawn regarding the structural aspects of such states from this study. The existence of the Free energy minima with respect to $\mathcal{P}$ demonstrates the thermodynamic preference of the amorphous state for a particular concentration of free volume corresponding to a specific density. That this brings a decrease in the Free energy of the structure shows that the metastability of the supercooled states is dependent upon the "free volume" present 
in the structure. The weakly localized states which correspond to the free energy minimum found in the region of small width parameter, although, do not gain stability by a significant percentage. This is reasonable since the presence of a small concentration of vacancies does not alter the density structure characterized by wide Gaussians. Thus the free energy does not change much although a clear minimum is observed with respect to the defect parameter $\mathcal{P}$ at a non-zero value of $\Delta_{v}$. At higher densities the stability of the highly localized states comes closer to that of the other and thus demonstrating that at higher densities, the supercooled state is likely to be characterized by the more localized density structures. We do not use here any of the more recent density functional theories like the MWDA ( modified weighted density approximation ) [20]. MWDA involves the global mapping of the inhomogeneous structure to a homogenous structure satisfying certain criteria in an effective medium type approximation. Thus it is unsatisfactory for systems characterized by local inhomogeneous effects like the presence of a small concentration of vacancies as is done here. Thus for qualitative investigation purpose, our use of RY functional approach is well justified in this study.

We would also like to indicate here that the major criticism of the results of the similar calculation done for the crystal structure [16], was the prediction of quite large values of $\Delta_{v}$. These were not in compliance with the experimental findings which predicted the defect densities in the crystal to be lesser than at least two orders of that predicted by Jones and Mohanty. In the present study, although we have used the mathematical formulation of the density function, its physical interpretation is entirely different. The defects in this study are a measure of the average amount of unoccupied volume present in the amorphous structure. Its concentration is actually indicative of the measure of dynamics possible in the system at a particular density. The vanishing of this free volume concentration is interpreted as a complete freezing of motion in the system and hence the relevance of this concept is not wavered by the critical value of $\Delta_{v}$ obtained. 
Since the free volume concentration is a measure of the transport properties in a system, qualitative indications can also be drawn with respect to the dynamical aspects. This study indicates that the decrease of the free volume can in general be associated with increase in viscosity of the system. The variation of free volume with density/temperature is useful in extracting the temperature dependence of the transport coefficients. We observe two different type of behavior for the two different free energy minima. The highly localized structures display a linear decrease in the free volume concentration with the increase in density. On the other hand, the weakly localized structures, characterized by the low value of the width parameter, show a power-law decrease in the free volume concentration with respect to density. It is well known that a large number of laboratory systems and computer simulations of simple systems show a power law divergence of viscosity with decrease in temperature. It is also worth considering here that the dynamical studies done using the Mode- Coupling Theories [21] also predict such a relation between the the transport properties and the density for low viscosity hard sphere systems. Thus this result suggests that the low $\alpha$ Free energy minima may correspond to the stationary state around which the fluctuations are considered in the dynamical studies coming under the realm of Mode-Coupling Theories. Although this would require further investigation and a definite relation to be established between the free volume concentration and the transport properties. 


\section{Figure Captions}

Fig 1: Difference in Free Energy per particle $(\Delta f)$ ( in units of $\beta^{-1}$ ) vs. $\mathcal{P}$ in the large $\alpha$ regime $\left(\rho_{o}{ }^{*}=1.12\right)$. In the inset the corresponding minimization is shown in the $\alpha$ space.

Fig 2 : Difference in Free Energy per particle $(\Delta f)$ ( in units of $\beta^{-1}$ ) vs. $\mathcal{P}$ in the low $\alpha$ regime $\left(\rho_{o}{ }^{*}=1.12\right)$. The inset shows the variation in the $\alpha$ space.

Fig 3: Free Volume concentration $\left(\Delta_{v}\right)$ vs. density $\rho_{o}{ }^{*}$ for the highly localized amorphous structure. The solid line depicts the linear fit.

Fig 4: Free Volume concentration $\left(\Delta_{v}\right)$ vs. density $\rho_{o}{ }^{*}$ for the weakly localized amorphous structure. The solid line depicts the power-law fit.

Fig 5: Difference in Free Energy per particle $(\Delta f)$ ( in units of $\beta^{-1}$ ) vs. $\rho_{o}$. The solid line corresponds to the small width parameter, $\alpha$, free energy minimum and the dashed line corresponds to the highly localized structure.

Fig 6: Variation of minimum $\alpha$ with the corresponding $\mathcal{P}$ at density $\rho_{o}=1.08$.

\section{References}

[1] M. H. Cohen and D. Turnbull, J. Chem. Phy. 31, 1164 (1959).

[2] Transport Theory and Stat. Phys., 24, Nos. 6-8 (1995), Special issue, edited by S. Yip and Paul Melman.

[3] Y.Singh, J.P.Stoessel and P.G.Wolynes, Phys. Rev. Lett., 541059 (1985). 
[4] G. Parisi Phys. Rev. Lett., 78, 4581 (1997).

[5] M. H. Cohen and G. S. Grest, Phys. Rev. B 20, 1077 (1979).

[6] G. Fox and P. J. Flory, J. Appl. Phys. 21, 581 (1950).

[7] A. K. Doolittle, J. Appl. Phys. 22, 1471 (1951).

[8] J. G. Kirkwood and E. Monroe, J. Chem. Phy. 8, 845 (1940).

[9] T. V. Ramakrishnan and M. Yussouff, Phys. Rev. B 19, 2775 (1979).

[10] A. D. J. Haymet and D. J. Oxtoby, J. Chem. Phys. 74, 2559 (1981).

[11] M.Baus and Jean-Louis Colot, J. Phys. C: Solid State Phys. 19, L135-L139(1986).

[12] Hartmut Löwen, J. Phys. C 2, 8477 (1990).

[13] C. Kaur and S. P. Das, Phys. Rev. Lett, 86, 2062.

[14] J. D. Bernal, Proc. Roy. Soc. London, Ser A 280, 299 (1964).

[15] P.Tarazona, Mol. Phys. 52, 871 (1984).

[16] G. L. Jones and U. Mohanty, Mol. Phys., 54, 1241 (1985).

[17] Charles Bennett, J. Appl. Phys, 43, 2727 (1972).

[18] L. Verlet and J. J. Weiss, Phys. Rev. 45, 939 (1972).

[19] D. Henderson and E. W. Grundke, J. Chem. Phys. 63, 601 (1975).

[20] A. R. Denton and N. W. Ashcroft, Phys. Rev. A, 39, 4701 (1989).

[21] S. P. Das, Phys. Rev. A, 36, 211 (1987) 


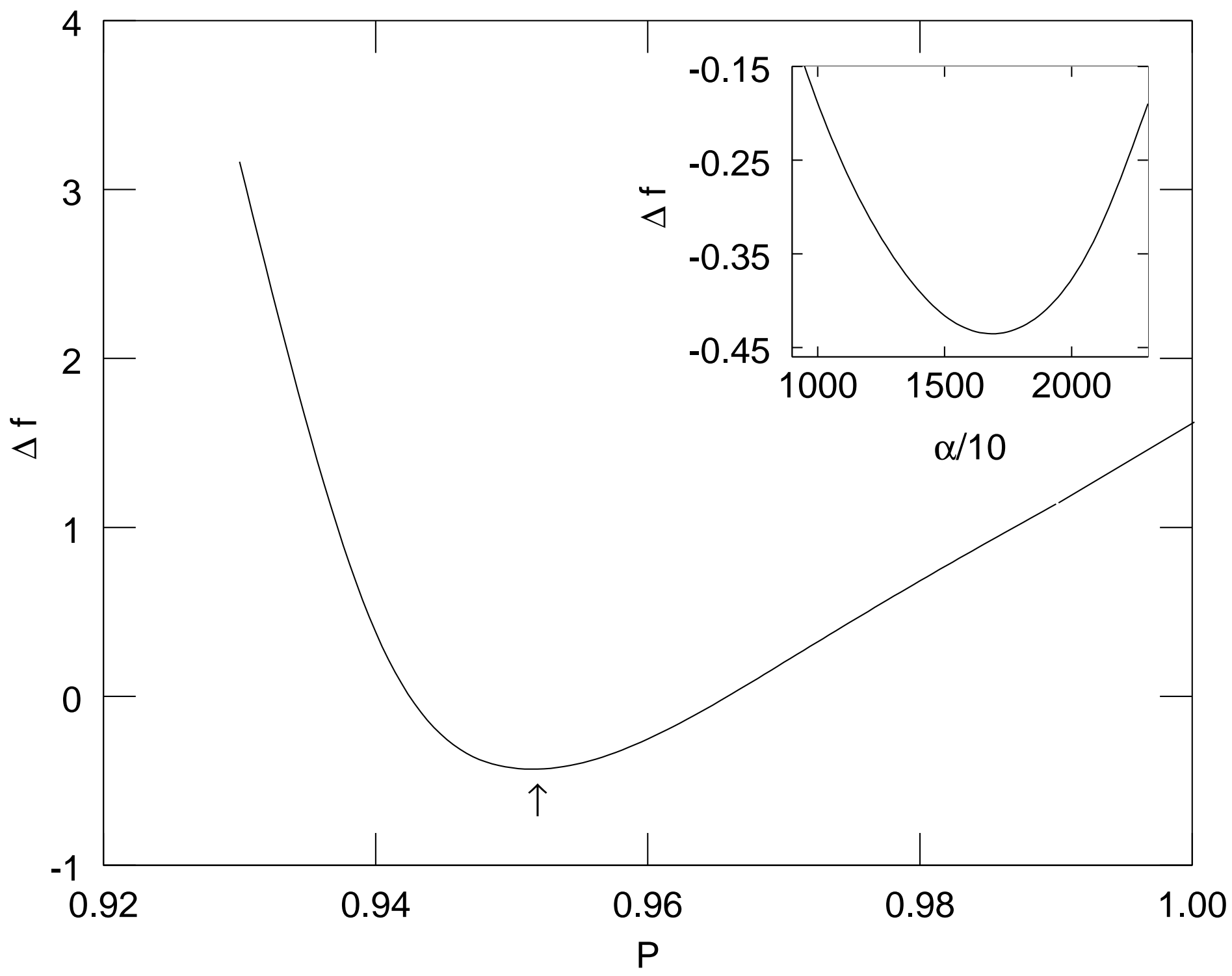




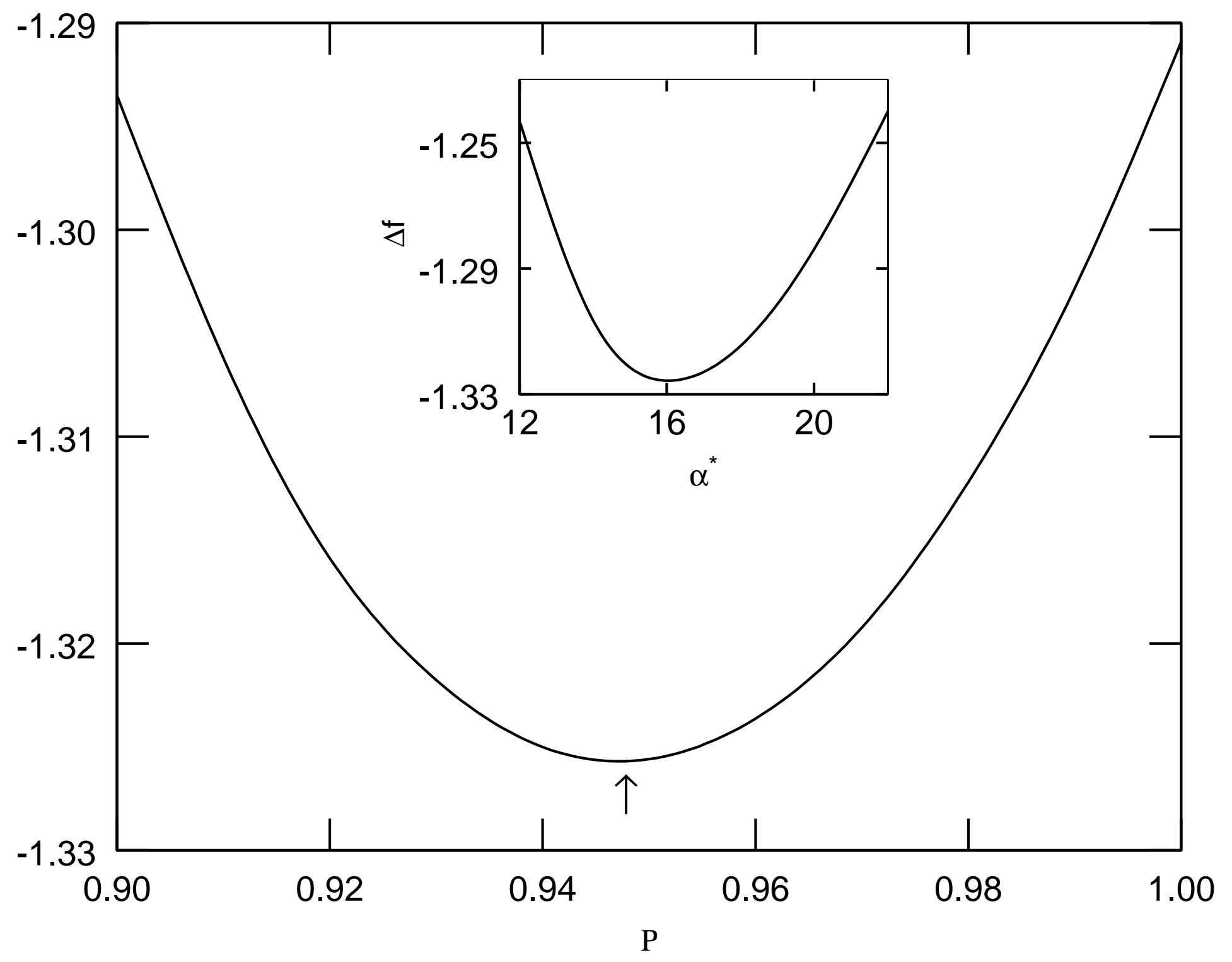




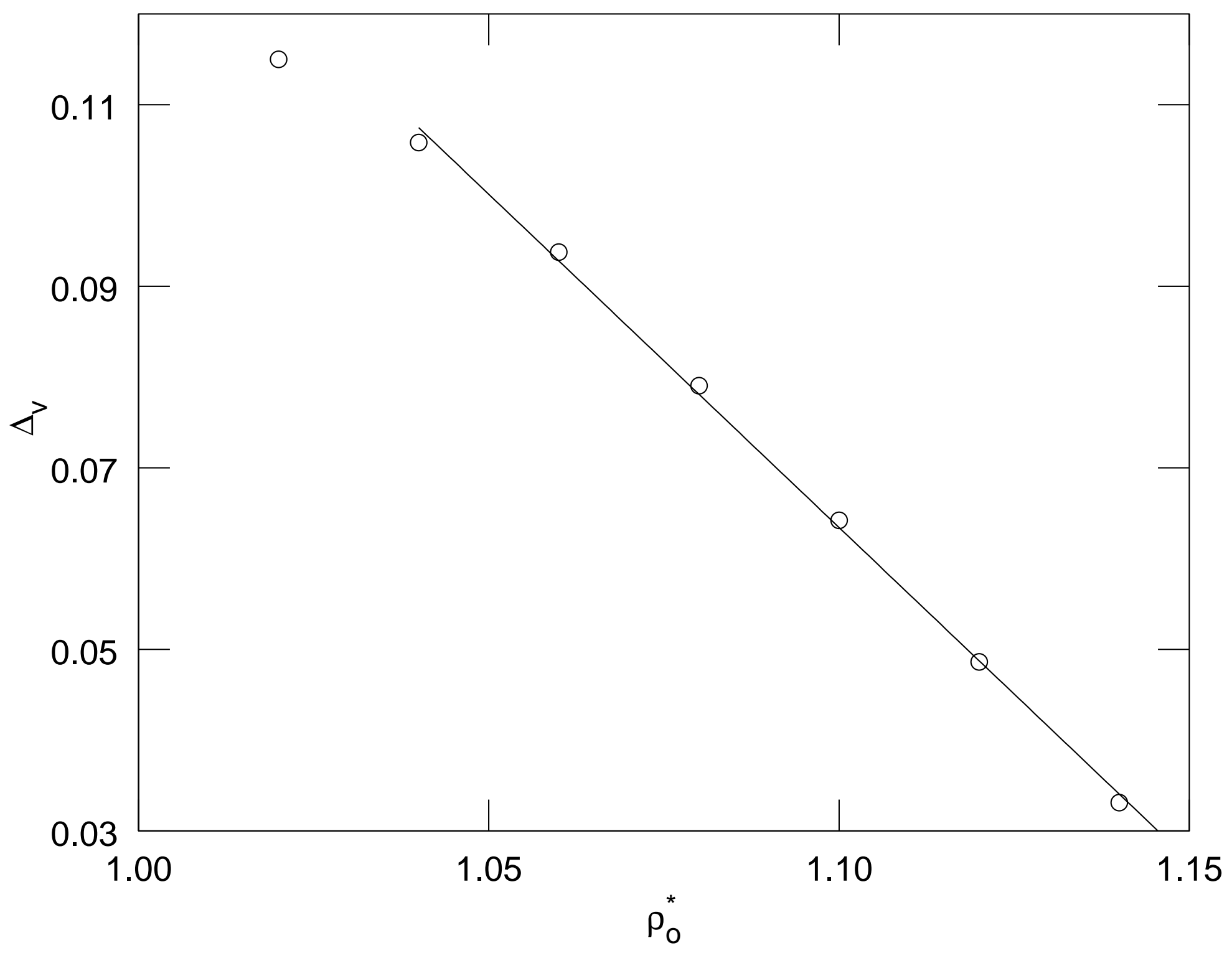




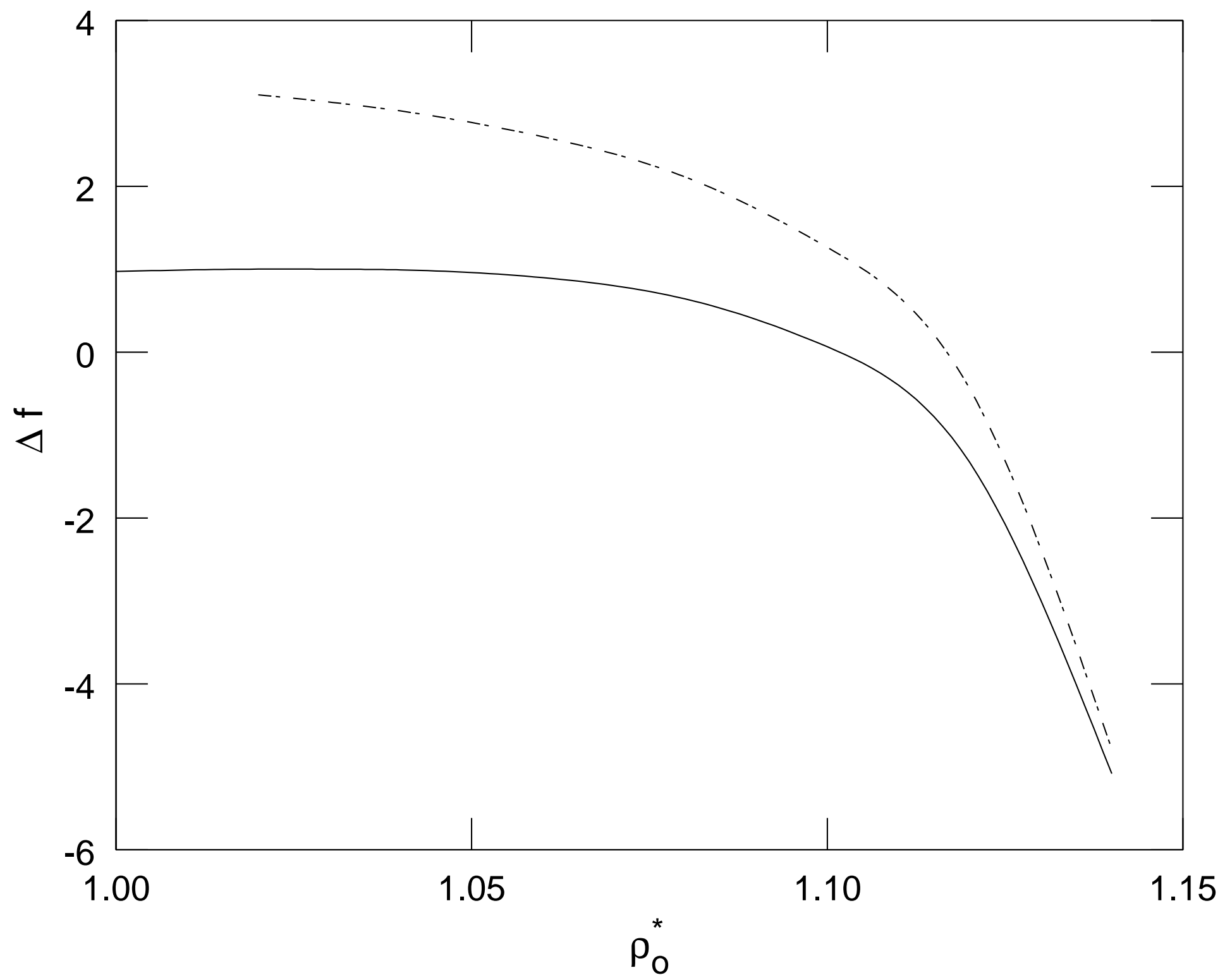




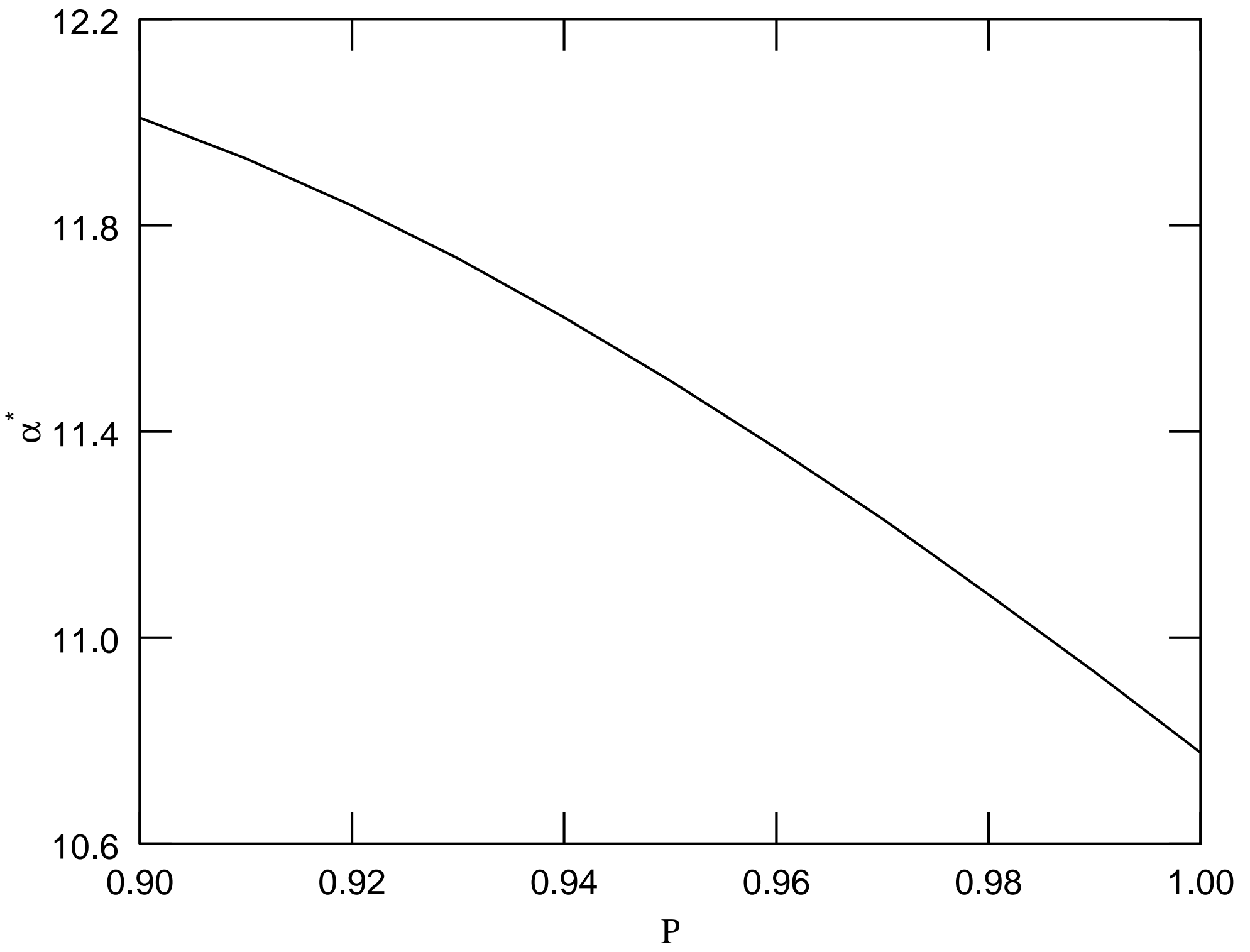

